\section{BMJ Open Respiratory Research}

\title{
Low utilisation of bronchoscopy to assess COVID-19 respiratory infection: a multicenter experience
}

Kamran Mahmood, ${ }^{1}$ Matt Abbott, ${ }^{1}$ Keriann Van Nostrand,${ }^{2}$ Rabih Bechara, ${ }^{3}$ Anne V Gonzalez, ${ }^{4}$ Amanda Brucker, ${ }^{5}$ Cynthia L Green, ${ }^{5}$ Christopher R Polage ${ }^{6}$

\section{ABSTRACT}

Abbott M, Van Nostrand K, et al. Low utilisation of bronchoscopy to assess COVID-19 respiratory infection: a multicenter experience. BMJ Open Resp Res 2021;8:e000962. doi:10.1136/ bmjresp-2021-000962

Received 16 April 2021 Accepted 5 July 2021

Check for updates

(C) Author(s) (or their employer(s)) 2021. Re-use permitted under CC BY-NC. No commercial re-use. See rights and permissions. Published by BMJ.

${ }^{1}$ Department of Medicine, Division of Pulmonary, Allergy and Critical Care, Duke University, Durham, North Carolina, USA

${ }^{2}$ Department of Medicine, Division of Pulmonary and Critical Care, Emory University, Atlanta, Georgia, USA

${ }^{3}$ Department of Medicine, Division of Pulmonary and Critical Care, Medical College of Georgia, Augusta, Georgia, USA

${ }^{4}$ Department of Medicine, Division of Pulmonary and Critical Care, McGill University, Montreal, Quebec, Canada

${ }^{5}$ Department of Biostatistics and Bioinformatics, Duke University School of Medicine, Durham, North Carolina, USA ${ }^{6}$ Department of Pathology, Duke University, Durham, North Carolina, USA

Correspondence to Dr Kamran Mahmood; k.mahmood@duke.edu respectively.
Objective For the diagnosis of COVID-19, the yield of nasopharyngeal (NP) swabs is unclear, and bronchoalveolar lavage (BAL) is obtained to confirm the diagnosis. We assessed the utilisation of bronchoscopy for COVID-19 diagnosis in a multicenter study and compared the diagnostic yield of BAL versus NP swabs.

Methods This retrospective study included all patients who were admitted with clinical presentation concerning for COVID-19 and underwent BAL from 1 March to 31 July 2020 at four tertiary care centres in North America. We also compared concordance of BAL with NP swabs for diagnosis of COVID-19 infection.

Results Fifty-three patients, with clinical suspicion for COVID-19 and admitted for respiratory failure, underwent bronchoscopy to collect BAL for SARS-CoV-2 testing. During the same period, 2039 bronchoscopies were performed on patients not infected with COVID-19. Of 42 patients with NP swabs and BAL collected within $\leq 7$ days, 1 was NP swab negative but positive by BAL for SARSCoV-2 $(n=1 / 42(2.4 \%))$. Across a wide array of testing platforms, the overall agreement between NP swabs and BAL results was $97.6 \%$ (95\% Cl: $93.0 \%$ to $100 \%$ ) with Cohen's k of 0.90 (95\% Cl: 0.69 to 1.00). The sensitivity, specificity, positive and negative predictive values of NP swabs compared with BAL were $83.3 \%$ (95\% Cl: $53.5 \%$ to $100 \%), 100 \%, 100 \%$ and $97.3 \%$ (95\% Cl: $92.1 \%$ to $100 \%)$,

Conclusions BAL was used infrequently to assess COVID-19 in busy institutions. NP swabs have a high concordance with BAL for COVID-19 testing, but negative NP swabs should be confirmed with BAL when clinical suspicion is high.

\section{INTRODUCTION}

The COVID-19 pandemic has led to devastating morbidity and mortality, primarily related to respiratory failure and associated multiorgan dysfunction. Prompt diagnosis of COVID-19 is critical for the treatment of infected individuals and prevention of infection transmission. The diagnosis of COVID-19 is established by clinical presentation, radiologic findings of the chest and testing based on reverse transcription (RT) PCR. The RT-PCR test is performed for the

\section{Key messages}

Bronchoscopies for bronchoalveolar lavage (BAL) represented $2.5 \%$ of total bronchoscopies performed in the participating institutions, and the overall agreement between nasopharyngeal (NP) swab and $\mathrm{BAL}$ results was significant at $97.6 \%(95 \% \mathrm{Cl}$ : $93.0 \%$ to $100 \%$ ).

We describe details of bronchoscopy practice and SARS-CoV-2 testing platforms at different institutions, and emphasize that when the suspicion for COVID-19 remains high despite negative NP swab, BAL should be performed to confirm the diagnosis.

detection of nucleic acid from SARS-CoV-2 on various specimens-nasopharyngeal (NP) swabs being one of the most common source of testing. ${ }^{12}$ However, several studies have reported that the sensitivity of NP swabs is variable. ${ }^{34}$ Since the COVID-19 infection involves the lower respiratory tract, especially in sicker patients, bronchoscopy is often performed in patients with negative NP swabs to obtain bronchoalveolar lavage (BAL), as an early study by Wang et al suggested that lower respiratory samples provide a higher diagnostic yield. ${ }^{4}$ In this study, the diagnostic yield of BAL was $93 \%$, while the yield of nasal swabs was only $63 \%$ from patients suspected of COVID-19. ${ }^{4}$ However, the bronchoscopy is an aerosol-generating procedure that can potentially expose the healthcare providers to COVID-19 and may strain the limited healthcare resources. ${ }^{5}$ Several professional societies came out early with guidelines advising against routine use of bronchoscopy for COVID-19 diagnosis, based on the experience from other coronavirus epidemics. ${ }^{6}$ The objectives of this study were to assess the clinical utilisation of bronchoscopy in COVID-19 diagnosis and compare the diagnostic yield of BAL and NP swabs in patients with suspected or confirmed COVID-19 infection. 


\section{METHODS}

\section{Study design and settings}

We performed a retrospective review of all patients who were clinically suspected to have COVID-19 and underwent BAL and NP swab testing for COVID-19 testing at four large academic institutions in North America. Medical records were reviewed for all patients who met the criteria from 1 March 2020 to 31 July 2020. Data from participating institutions were entered into a REDCap database maintained by Duke University.

\section{Patient and public involvement statement}

It was not appropriate or possible to involve patients or the public in the design, or conduct, or reporting, or dissemination plans of our research, but the study was performed to improve patient care outcomes. We thank our patients and their families for their strength and endurance, as stated in the Acknowledgements.

\section{Specimen collection}

NP swabs were collected by trained healthcare personnel, according to Centers for Disease Control and Prevention (CDC) recommendations. ${ }^{78}$ The swabs were made of synthetic fibre attached to a plastic or aluminium shaft. The swabs were inserted in the posterior nasopharynx, parallel to the palate and maintained in place for a few seconds to absorb the secretions. Afterwards, the swabs were removed and placed immediately into a sterile transport tube containing $2-3 \mathrm{~mL}$ of viral transport medium or sterile saline. NP swabs for point-of-care nucleic acid amplification (NAA) tests were performed directly on the instrument near the collection location.

BAL was performed via bronchoscopy by trained healthcare providers. The bronchoscope was inserted into the airways and wedged into a distal, segmental bronchus. Then, sterile saline in two to three $50 \mathrm{~mL}$ aliquots was instilled into the airway and suctioned back into a trap. From the retrieved BAL fluid, 2-3 mL was sent in a sterile container for SARS-CoV-2 RT-PCR.

\section{COVID testing}

SARS-CoV-2 coronavirus testing was performed following the manufacturer's instructions for use and/or validated protocols on multiple US Food and Drug Administration emergency use authorisation RT-PCR or other NAA test platforms. Tests were independently verified and validated by each performing laboratory before clinical use. NP samples were generally tested on a laboratory RT-PCR assay (Cepheid Xpert SARS-CoV-2, DiaSorin Simplexa COVID-19 Direct, Roche cobas SARS-CoV-2 and Panther Fusion SARS-CoV-2), with a few tested on a rapid point-ofcare NAA test (Abbott ID Now COVID-19). BAL samples were only tested by laboratory RT-PCR on platforms validated locally for BAL (CDC SARS-CoV-2, Abbott Alinity m SARS-CoV-2, Cepheid Xpert SARS-CoV-2, Roche cobas SARS-CoV-2 and Panther Fusion SARS-CoV-2).
Statistical analysis

Continuous variables are presented as median with 25th and 75th percentiles (Q1-Q3), and categorical variables as frequency counts with percentages. The concordance of NP swab and BAL testing was assessed using McNemar's test and Cohen's $k$ with 95\% CI. A two-sided $p$ value of 0.05 or less was considered statistically significant. All analyses were performed with SAS V.9.4 (SAS Institute).

\section{RESULTS}

Bronchoscopic BAL was performed to assess for COVID-19 infection on 53 hospitalised patients at four centres during the study period, while 2183 patients were admitted with confirmed COVID-19 diagnosis. Overall, 2039 bronchoscopies were performed on patients not infected with COVID-19 during this time frame. COVID-19 was confirmed in 13 of our cohort of 53 patients who underwent BAL. Confirmed patients with COVID-19 $(\mathrm{N}=13)$ underwent a total of 57 non-bronchoscopy procedures and 2 patients had a repeat bronchoscopy performed; bronchoscopies thus accounted for $26.3 \%$ of total procedures.

The clinical characteristics of the patients included in the study are summarised in table 1 . The patients included in the study were deemed high risk for COVID-19 as they had symptoms and radiographic findings concerning for COVID-19 infection along with respiratory failure or acute respiratory distress syndrome (ARDS). Forty-six patients $(86.8 \%)$ were admitted in intensive care units (ICU), while others were admitted on medical wards. Diabetes mellitus $(32.1 \%)$ was the most common comorbid condition. Chest radiographs showed bilateral infiltrates in $83 \%$, unilateral infiltrates in $11.3 \%$ and pleural effusions in $15.1 \%$ of patients. Chest CT scan showed consolidation in $43.4 \%$, ground-glass infiltrates in $58.5 \%$, interlobular septal thickening in 3.8\%, mediastinal lymphadenopathy in $3.8 \%$, and pleural effusions in $26.4 \%$ of patients. Forty-six patients $(86.8 \%)$ were on mechanical ventilation; 31 patients $(59.5 \%)$ had ARDS and 5 patients $(9.4 \%)$ required extracorporeal membrane oxygenation.

Prior to BAL, 41 patients had negative NP swabs, while 12 patients had positive NP swabs for SARS-CoV-2 testing. BAL was done in patients with negative NP swabs to confirm the COVID-19 diagnosis, while the BAL in positive NP swab patients was performed to assess for other coinfections. In the patients $(\mathrm{N}=13)$ who were later confirmed to have COVID-19, the BAL was performed at a median of 14 days (Q1-Q3: 8-18 days) from the onset of symptoms. In 46 patients $(86.8 \%)$, the last NP swab closest to BAL was done when they were on mechanical ventilation, and 7 patients (13.2\%) had swabs obtained while spontaneously breathing. A wide array of platforms was used for COVID-19 testing on NP swabs and BAL, as shown in table 2. Some patients had multiple negative NP swabs done before BAL was performed.

All bronchoscopies were done in a negative pressure room with powered air purifying respirator or N95 with 


\begin{tabular}{|c|c|}
\hline Baseline characteristics & $\begin{array}{l}\text { Total } \\
(\mathrm{N}=53)\end{array}$ \\
\hline Age, median years (Q1-Q3) & $62(46-69)$ \\
\hline \multicolumn{2}{|l|}{ Gender, n (\%) } \\
\hline Male & $36(67.9)$ \\
\hline Female & $17(32.1)$ \\
\hline \multicolumn{2}{|l|}{ Race, n (\%) } \\
\hline African American & $16(30.2)$ \\
\hline Asian & $2(3.8)$ \\
\hline Caucasian & $30(56.6)$ \\
\hline Hispanic & $1(1.9)$ \\
\hline Other & $4(7.5)$ \\
\hline BMI, median kg/m² (Q1-Q3) & $28(23.7-34.2)$ \\
\hline \multicolumn{2}{|l|}{ Comorbidities, n (\%) } \\
\hline Diabetes & $17(32.1)$ \\
\hline Congestive heart failure & $9(17.0)$ \\
\hline Coronary artery disease & $13(24.5)$ \\
\hline Hypertension & $14(26.4)$ \\
\hline Cirrhosis/liver failure & $4(7.5)$ \\
\hline Chronic kidney disease/renal failure & $12(22.6)$ \\
\hline Thrombocytopenia & $4(7.5)$ \\
\hline Malignancy & $8(15.1)$ \\
\hline Lung transplant & 7 (13.2) \\
\hline $\begin{array}{l}\text { Chronic obstructive pulmonary } \\
\text { disease }\end{array}$ & $7(13.2)$ \\
\hline \multicolumn{2}{|l|}{ COVID-19 symptoms, n (\%) } \\
\hline Cough & $28(52.8)$ \\
\hline Shortness of breath & $40(75.5)$ \\
\hline Fever & $20(37.7)$ \\
\hline Hypoxaemia & $31(58.5)$ \\
\hline Chest pain & $1(1.9)$ \\
\hline Haemoptysis & $2(3.8)$ \\
\hline Fatigue & $3(5.7)$ \\
\hline Gastrointestinal symptoms & $2(3.8)$ \\
\hline
\end{tabular}

$X$-ray findings of the chest infected with COVID-19, n (\%)

\begin{tabular}{ll} 
Bilateral infiltrates & $44(83.0)$ \\
Unilateral infiltrates & $6(11.3)$ \\
Pleural effusion & $8(15.1)$ \\
$\begin{array}{l}\text { CT findings of the chest infected with } \\
\text { COVID-19, } \mathrm{n}(\%)\end{array}$ & \\
Consolidation & $23(43.4)$ \\
Ground-glass infiltrates & $31(58.5)$ \\
Interlobular septal thickening & $2(3.8)$ \\
Mediastinal lymphadenopathy & $2(3.8)$ \\
Pleural effusion & $14(26.4)$ \\
Not done & $2(3.8)$ \\
\hline
\end{tabular}

Continued

\begin{tabular}{|c|c|}
\hline Baseline characteristics & $\begin{array}{l}\text { Total } \\
(\mathrm{N}=53)\end{array}$ \\
\hline \multicolumn{2}{|l|}{ ARDS, n (\%) } \\
\hline No & $22(41.5)$ \\
\hline Mild & $10(18.9)$ \\
\hline Moderate & $15(28.3)$ \\
\hline Severe & $6(11.3)$ \\
\hline $\begin{array}{l}\mathrm{FiO}_{2} \text { - baseline at the time of } \\
\text { bronchoscopy, median (Q1-Q3) }\end{array}$ & $0.40(0.30-0.50)$ \\
\hline $\begin{array}{l}\mathrm{PaO}_{2} \text { - baseline at the time of } \\
\text { bronchoscopy, median mmHg (Q1-Q3) }\end{array}$ & $\begin{array}{l}n=43 \\
90(72-115)\end{array}$ \\
\hline ECMO, n (\%) & $5(9.4)$ \\
\hline $\begin{array}{l}\text { Respiratory failure requiring mechanical } \\
\text { ventilation, } \mathrm{n}(\%)\end{array}$ & $46(86.8)$ \\
\hline Ventilator mode, $\mathrm{n}(\%)$ & $\mathrm{n}=46$ \\
\hline Pressure control & $20(43.5)$ \\
\hline Pressure support & $16(34.8)$ \\
\hline Volume control & $10(21.7)$ \\
\hline $\begin{array}{l}\text { Driving pressure for pressure control, } \\
\text { median } \mathrm{cm} \mathrm{H}_{2} \mathrm{O}(\mathrm{Q} 1-\mathrm{Q} 3)\end{array}$ & $\begin{array}{l}n=20 / 46 \\
16(11-18)\end{array}$ \\
\hline Driving volume, median $\mathrm{mL}$ (Q1-Q3) & $\begin{array}{l}n=10 / 46 \\
390(350-450)\end{array}$ \\
\hline PEEP, median $\mathrm{cm} \mathrm{H}_{2} \mathrm{O}$ (Q1-Q3) & $\begin{array}{l}n=46 \\
12(8-14)\end{array}$ \\
\hline
\end{tabular}

*ARDS defined according to Berlin definition where $\mathrm{PaO}_{2} / \mathrm{FiO}_{2}$ ratio of $201-300$ is mild, $101-200$ is moderate and $\leq 100$ is severe ARDS.

.ARDS, acute respiratory distress syndrome; BMI, body mass index; ECMO, extracorporeal membrane oxygenation; $\mathrm{FiO}_{2}$, fractional inspired oxygen; $\mathrm{PaO}_{2}$, arterial oxygen tension ; PEEP, positive end-expiratory pressure.

face shields (table 3). Most bronchoscopies (96.2\%) were done with deep sedation or general anaesthesia. Endotracheal tube $(92.2 \%)$ was the preferred airway device for bronchoscopy. Disposable bronchoscopes were used for $36(67.9 \%)$ procedures. The bronchoscopies were tolerated well and only one patient with septic shock developed transient hypotension requiring temporary escalation of vasopressors.

Across different platforms at the participating institutions, the concordance between NP swabs and BAL was evaluated in 42 patients who had NP swabs and BAL collected within 7 days. The NP swabs closest to BAL were used for concordance assessment, and were collected at a median of 1 day (Q1-Q3: 0-4 days) prior to BAL. One patient had a negative NP swab but subsequent BAL was positive $(\mathrm{n}=1 / 42(2.4 \%)$ table 4$)$; the remaining 41 patients had NP swabs and BAL results that were concordant, yielding an overall agreement of $97.6 \%$ (95\% CI: $93.0 \%$ to $100 \%$ ) and Cohen's $\mathrm{k}$ of 0.90 (95\% CI: 0.69 to 1.00$)$. In addition, the $\mathrm{p}$ value from McNemar's test for marginal homogeneity was 0.317 ; therefore, the null hypothesis that the NP swabs and BAL were concordant 
Table 2 COVID-19 and other diagnostic testing for patients with NP swabs and BAL performed within 7 days

\begin{tabular}{ll}
\hline CoVID-19 and other testing characteristics & $\begin{array}{l}\text { Total } \\
\mathbf{( N = 4 2 )}\end{array}$ \\
\hline $\begin{array}{l}\text { No. of NP swabs performed } \\
\text { Total }\end{array}$ & 82 \\
Within 1 week and closest to BAL & 42
\end{tabular}

No. of patients with single or multiple NP swabs within 7 days of BAL

$\begin{array}{ll}1 & 14(33.3) \\ 2 & 16(38.1) \\ \geq 3 & 12(28.6)\end{array}$

NP swab testing platforms (\%) within 1 week and closest to BAL

\begin{tabular}{|c|c|}
\hline Cepheid Xpert & $24(57.1)$ \\
\hline DiaSorin & $8(19.0)$ \\
\hline Abbott ID Now & $3(7.1)$ \\
\hline Roche cobas 6800 & $4(9.5)$ \\
\hline Panther Fusion & $1(2.3)$ \\
\hline Missing (other institution) & $2(4.8)$ \\
\hline \multicolumn{2}{|l|}{ No. of BAL performed for COVID-19 testing } \\
\hline Total & 53 \\
\hline Within 7 days of NP swab & 42 \\
\hline \multicolumn{2}{|l|}{ BAL testing platforms within 7 days of NP swab (\%) } \\
\hline Abbott Alinity & $1(2.4)$ \\
\hline Centers for Disease Control and Prevention & $26(61.9)$ \\
\hline Cepheid Xpert & $7(16.7)$ \\
\hline Roche cobas 6800 & $6(14.3)$ \\
\hline Panther Fusion & $2(4.8)$ \\
\hline $\begin{array}{l}\text { Days between symptom onset and BAL in } \\
\text { COVID-19 confirmed patients, median (Q1-Q3) }\end{array}$ & $\begin{array}{l}n=13 \\
14(8-18)\end{array}$ \\
\hline $\begin{array}{l}\text { Days between NP swab and BAL performed within } \\
7 \text { days, median (Q1-Q3) }\end{array}$ & $1(0-4)$ \\
\hline \multicolumn{2}{|l|}{ Positive BAL cultures other than COVID-19 } \\
\hline Bacterial, n (\%) & $3(7.1)$ \\
\hline Staphylococcus aureus & $2(4.8)$ \\
\hline Pseudomonas aeruginosa & $1(2.4)$ \\
\hline Fungal, $\mathrm{n}(\%)-$ Aspergillus fumigatus & $1(2.4)$ \\
\hline Acid fast bacilli, $\mathrm{n}(\%)-$ Mycobacterium avium & $1(2.4)$ \\
\hline Pneumocystis jirovecii PCR, n (\%) & $1(2.4)$ \\
\hline
\end{tabular}

BAL, bronchoalveolar lavage; NP, nasopharyngeal.

could not be rejected. The sensitivity, specificity, positive predictive value and negative predictive value of NP swabs compared with BAL were $83.3 \%$ (95\% CI: $53.5 \%$ to $100 \%$ ), $100 \%, 100 \%$ and $97.3 \%$ (95\% CI: $92.1 \%$ to $100 \%)$, respectively.

\section{DISCUSSION}

This multicenter study evaluated the clinical utilisation of bronchoscopy for COVID-19 diagnosis and showed that bronchoscopy and BAL for diagnosis of COVID-19 made

\begin{tabular}{ll}
\hline Table 3 Bronchoscopy data & \\
\hline Bronchoscopy characteristics & Total (N=53) \\
\hline Sedation, $\mathrm{n}(\%)$ & $51(96.2)$ \\
\hline Deep/general & $1(1.9)$ \\
\hline Moderate & $1(1.9)$ \\
\hline Awake/local anaesthetic & $47(92.2)$ \\
\hline Airway for deep sedation/general anaesthesia, $\mathrm{n}(\%)$ \\
\hline Endotracheal tube & $4(7.8)$ \\
\hline Laryngeal mask airway & \\
\hline Bronchoscope type, $\mathrm{n}$ (\%) & $36(67.9)$ \\
\hline Disposable & $17(32.1)$ \\
\hline Non-disposable & $40(75.5)$ \\
\hline Type of respiratory protective equipment, $\mathrm{n}(\%)$ \\
\hline Powered air purifying respirator & $13(24.5)$ \\
\hline N95 with face shield & $53(100)$ \\
\hline Negative pressure room, $\mathrm{n}$ (\%) & $21(39.6)$ \\
\hline Medications while performing bronchoscopy, $\mathrm{n}(\%)$ \\
\hline Aspirin & $2(3.8)$ \\
\hline Clopidogrel & $11(20.8)$ \\
\hline Heparin-subcutaneous & 0 \\
\hline Heparin-intravenous & $10(18.9)$ \\
\hline Enoxaparin-prophylactic & 0 \\
\hline Other anticoagulants & $21(43.8)$ \\
\hline Vasopressors & $1(1.8)$ \\
\hline Complications, $\mathrm{n}$ (\%) & \\
\hline Hypotension & \\
\hline
\end{tabular}

up a small fraction of overall bronchoscopies in busy US and Canadian centres. The high concordance between $\mathrm{NP}$ swabs and BAL is reassuring, but BAL should be considered in patients with negative NP swabs who have a high clinical suspicion for COVID-19 infection.

The participating centres in the study were tertiary care, academic medical centres with a high volume of bronchoscopic procedures and patients with COVID19. However, bronchoscopy and BAL utilisation was low, accounting for only about $2.5 \%$ of total bronchoscopies, and NP swabs were primarily used to assess COVID-19. This practice is in accordance with the guidelines issued by American College of Chest Physicians (ACCP) and

Table 4 NP swab* and BAL test results performed within 7 days

\begin{tabular}{cllcc} 
& & \multicolumn{3}{l}{ BAL result } \\
\cline { 3 - 5 } & Frequency & Positive & Negative & Total \\
\hline \multirow{2}{*}{ NP swab result } & Positive & 5 & 0 & 5 \\
& Negative & 1 & 36 & 37 \\
& Total & 6 & 36 & 42 \\
\hline
\end{tabular}

*NP swabs collected closest to BAL were used for the analysis. BAL, bronchoalveolar lavage; NP, nasopharyngeal. 
American Association of Bronchology and Interventional Pulmonology, based on experience from previous coronavirus epidemics, and aimed to prevent the exposure to healthcare workers. ${ }^{6}$

We found a high concordance rate between NP swabs and BAL. One of the original reports describing the diagnostic yield of respiratory specimens was reported by Wang et al. ${ }^{4}$ They described the diagnostic yield of 8 nasal swabs, 398 pharyngeal swabs and 15 BAL specimens in patients admitted with COVID-19 infection. The positive test rate from nasal swabs, pharyngeal swabs and BAL was $63 \%, 32 \%$ and $93 \%$, respectively. Gao et al reported their single-centre experience in 123 patients with COVID-19 infection and respiratory failure requiring mechanical ventilation. ${ }^{9}$ Fourteen patients $(11 \%)$ had discordant NP swabs and BAL assays. When compared with BAL, they described the sensitivity of the NP swab to be $88.6 \%$, specificity $88.6 \%$, positive predictive value $93.3 \%$, negative predictive value $81.3 \%$ and accuracy of $88.6 \%$. In another study, Geri et al evaluated the agreement between negative NP swabs and subsequent BAL in 79 patients admitted with respiratory failure. ${ }^{10}$ Two patients with negative NP swabs had positive BAL with an accuracy of $97.5 \%$ (Cohen's k=0.487). In another study, in 28 patients with suspected COVID-19, 3 sequential negative NP swabs, and negative IgG and IgM serologies were completely concordant with negative BAL results. ${ }^{11}$ Barberi et al reported in a cohort of 198 patients with suspected COVID-19 and negative NP swabs, 32 (16\%) patients had positive BAL. ${ }^{12}$ But multiple case reports and studies have reported lower concordance between NP swabs and BAL. ${ }^{13-15}$ Patrucco et al reported a sensitivity of NP swabs of $23 \%$ in a cohort of 43 patients, as 33 patients with negative NP swabs were subsequently diagnosed with COVID-19 on BAL. ${ }^{16}$ Similarly, Mondoni et al reported a sensitivity of NP swabs of only $44.8 \%$ in their series, as 43 out of 78 patients with negative NP swabs tested positive for COVID-19 on BAL. ${ }^{17}$ Therefore, the published literature about the yield and concordance of NP swabs and BAL is highly variable. We found a high concordance of $97.6 \%$ (Cohen's $\mathrm{k}=0.90$ ) between NP swabs and BAL with a wide range of testing platforms. Our results are more aligned with published studies of high concordance, which might be related to a better technique of NP swab collection-a critical step stressed by many experts. ${ }^{18} 19$ The high agreement between NP swabs and BAL and attempt to minimise provider exposure to aerosol-generating bronchoscopy may be the reason why BAL was performed infrequently to diagnose COVID-19 at our institutions.

COVID-19 primarily affects the lower respiratory tract, especially in patients with severe disease, and thus lower respiratory specimens such as BAL are expected to have a higher diagnostic yield. ${ }^{20}$ There are reports of initial negative upper respiratory RT-PCR tests in patients with clinical or CT scan findings consistent with COVID-19, but subsequent upper respiratory samples were positive on repeat testing. ${ }^{21}{ }^{22}$ In addition, NP swabs may be fraught with suboptimal specimen collection technique..$^{18}$ In a report of four patients with suggestive symptoms and negative NP swab, repeat NP swabs by otolaryngologists within hours were positive. ${ }^{19}$ All the patients had nasal obstruction, and the initial false-negative test was attributed to inadequate sampling. Another study also found lower human DNA on suspected false-negative NP swabs, suggestive of suboptimal sampling. ${ }^{23}$ The high yield and concordance of NP swabs with BAL in some published studies and our cohort highlights this important diagnostic consideration.

Radiological findings suggestive of COVID-19 infection might be helpful but have low sensitivity and specificity, as in a study evaluating chest CT based COVID-19 probability scores in negative NP swab patients, 7/46 (15\%) patients with atypical or low suspicion CT scans had positive BAL. ${ }^{24}$ Similarly, in another study evaluating 50 patients suspected of COVID-19 with negative NP swabs, 3 patients with CT scan indeterminate for COVID-19 were found to have positive BAL. ${ }^{15}$ Therefore, $\mathrm{WHO}$ and ACCP guidelines suggest obtaining a lower respiratory specimen if the upper respiratory specimen is negative and clinical suspicion of COVID-19 is high. ${ }^{62}$ Our study findings support the available data and expert opinion, and in patients who remain suspicious for COVID-19 infection despite NP swab testing and radiographic findings, BAL testing should be performed to confirm the diagnosis.

Bronchoscopy may be necessary to establish alternate diagnosis in patients with suspected COVID-19 infection. Torrego et al reported their experience of bronchoscopy in 101 patients with COVID-19 on mechanical ventilation. ${ }^{26}$ They reported presence of thick secretions and studies positive for other pathogens in $29 \%$ of the patients. In our cohort of patients who had NP swab and BAL done concomitantly, the BAL studies isolated other pathogens in $14.2 \%(6 / 42)$ patients. The lower isolation of other pathogens in our study could be related to differences in underlying disease process or ongoing antibiotic coverage.

\section{Strengths and limitations}

The strengths of this study include its multicenter design that provides a broader picture of the bronchoscopy practice for diagnosis of COVID-19 in North America compared with the previous single-centre studies and suggests that the negative predictive value of a wellcollected NP swab for a subsequent negative BAL may be higher than previous reports. The study also evaluated a broad spectrum of platforms for COVID-19 testing on NP swabs and BAL. The weaknesses of the study are its retrospective design and limited number of patients who had BAL specimens available for comparison with NP swabs, which is in alignment with current guidelines. During the COVID-19 pandemic, bronchoscopies for non-COVID-19 indications continued at our institutions. We instituted a policy to obtain a preprocedure NP swab for COVID-19 
testing, and the bronchoscopies were performed only if NP swabs were negative. Since BAL was not performed to assess for COVID-19 during these bronchoscopies, we cannot comment on the value of BAL in asymptomatic patients for COVID-19.

\section{CONCLUSIONS}

In conclusion, this multicenter experience suggests a high concordance between NP swabs and BAL for diagnosis of COVID-19 in patients with severe acute lower respiratory illness and a high negative predictive value of NP swabs for subsequent negative BAL. This experience supports professional societal recommendations limiting bronchoscopy procedures in persons under investigation for COVID-19 and may have contributed to a decreased utilisation of bronchoscopy for diagnosis of COVID-19 at these centres. However, NP swab results should be interpreted in clinical and epidemiological context, and BAL testing should be considered to definitively rule out COVID-19 in patients with negative NP swabs when clinical suspicion of COVID-19 remains high.

Acknowledgements We acknowledge and thank all the healthcare workers for their dedication, the patients infected with COVID-19 and their families for their strength and endurance. We additionally wish to acknowledge support from the Biostatistics, Epidemiology and Research Design Methods Core funded through Grant Award Number UL1TR002553 from the National Center for Advancing Translational Sciences, a component of the National Institutes of Health (NIH). The content is solely the responsibility of the authors and does not necessarily represent the official views of the $\mathrm{NIH}$.

Contributors KM wrote the manuscript which was reviewed, edited and approved by all authors. KM, MA, KMVN, RB, AVG and CRP designed the study and collected the data. $A B$ and $C G$ analysed the data. KM took full responsibility for the integrity of the manuscript.

Funding The authors have not declared a specific grant for this research from any funding agency in the public, commercial or not-for-profit sectors.

\section{Competing interests None declared.}

Patient and public involvement Patients and/or the public were not involved in the design, or conduct, or reporting, or dissemination plans of this research.

Patient consent for publication Not required.

Ethics approval Institutional review boards (IRB) of all the institutions, including Duke University (IRB no. 00106066), Emory University, Medical College of Georgia and McGill University approved the study.

Provenance and peer review Not commissioned; externally peer reviewed.

Data availability statement Data are available upon reasonable request.

Open access This is an open access article distributed in accordance with the Creative Commons Attribution Non Commercial (CC BY-NC 4.0) license, which permits others to distribute, remix, adapt, build upon this work non-commercially, and license their derivative works on different terms, provided the original work is properly cited, appropriate credit is given, any changes made indicated, and the use is non-commercial. See: http://creativecommons.org/licenses/by-nc/4.0/.

\section{REFERENCES}

1 Wang D, Hu B, Hu C, et al. Clinical characteristics of 138 hospitalized patients with 2019 novel coronavirus-infected pneumonia in Wuhan, China. JAMA 2020;323:1061

2 Mohammadi A, Esmaeilzadeh E, Li Y, et al. SARS-CoV-2 detection in different respiratory sites: a systematic review and meta-analysis. medRxiv 2020

3 Woloshin S, Patel N, Kesselheim AS. False negative tests for SARS-CoV-2 infection - challenges and implications. N Engl J Med 2020;383:e38.
4 Wang W, Xu Y, Gao R, et al. Detection of SARS-CoV-2 in different types of clinical specimens. JAMA 2020;2.

5 Fink JB, Ehrmann S, Li J. Reducing aerosol-related risk of transmission in the era of COVID-19: an interim guidance endorsed by the International Society of aerosols in medicine. J Aerosol Med Pulm Drug Deliv 2020;33:300-4

6 Wahidi MM, Shojaee S, Lamb CR, et al. The use of bronchoscopy during the coronavirus disease 2019 pandemic: CHEST/AABIP guideline and expert panel report. Chest 2020;158:1268-81.

7 Marty FM, Chen K, Verrill KA. How to obtain a nasopharyngeal swab specimen. reply. N Engl J Med 2020;383:e14.

8 Mahmood K. You Can't Improve What You Can't Measure: Smart Learning Meets Rigid Bronchoscopy. J Bronchology Interv Pulmonol 2020;27:227-78 https://www.cdc.gov/coronavirus/2019-nCoV/ lab/guidelines-clinical-specimens.html\#specimen. Accessed $12 / 15 / 2020$.

9 Gao C, Cuttica M, Malsin E, et al. Comparing nasopharyngeal and bronchoalveolar lavage SARS-CoV-2 assays in respiratory failure. Am J Respir Crit Care Med 2020.

10 Geri P, Salton F, Zuccatosta L, et al. Limited role for bronchoalveolar lavage to exclude COVID-19 after negative upper respiratory tract swabs: a multicentre study. Eur Respir J 2020;56. doi:10.1183/13993003.01733-2020. [Epub ahead of print: 08 Oct 2020].

11 Ora J, Puxeddu E, Cavalli F, et al. Does bronchoscopy help the diagnosis in COVID-19 infection? Eur Respir J 2020;56. doi:10.1183/13993003.01619-2020. [Epub ahead of print: 06 Aug 2020].

12 Barberi C, Castelnuovo E, Dipasquale A, et al. Bronchoalveolar lavage in suspected COVID-19 cases with a negative nasopharyngeal swab: a retrospective cross-sectional study in a high-impact northern Italy area. Intern Emerg Med 2021;12.

13 Gualano G, Musso M, Mosti S, et al. Usefulness of bronchoalveolar lavage in the management of patients presenting with lung infiltrates and suspect COVID-19-associated pneumonia: a case report. Int $J$ Infect Dis 2020;97:174-6.

14 Ramos KJ, Kapnadak SG, Collins BF, et al. Detection of SARSCoV-2 by bronchoscopy after negative nasopharyngeal testing: stay vigilant for COVID-19. Respir Med Case Rep 2020;30:101120.

15 Yserbyt J, Wauters E, Testelmans D. The role of flexible bronchoscopy in Swab-negative patients during the SARS-CoV2 pandemic. J Bronchology Interv Pulmonol 2020.

16 Patrucco F, Albera C, Bellocchia M, et al. SARS-CoV-2 detection on bronchoalveolar lavage: an Italian multicenter experience. Respiration 2020;99:970-8.

17 Mondoni M, Sferrazza Papa GF, Rinaldo R, et al. Utility and safety of bronchoscopy during the SARS-CoV-2 outbreak in Italy: a retrospective, multicentre study. Eur Respir J 2020;56. doi:10.1183/13993003.02767-2020. [Epub ahead of print: 15 Oct 2020]

18 Lippi G, Simundic A-M, Plebani M. Potential preanalytical and analytical vulnerabilities in the laboratory diagnosis of coronavirus disease 2019 (COVID-19). Clin Chem Lab Med 2020;58:1070-6.

19 Piras A, Rizzo D, Uzzau S, et al. Inappropriate nasopharyngeal sampling for SARS-CoV-2 detection is a relevant cause of falsenegative reports. Otolaryngol Head Neck Surg 2020;163:459-61.

20 Branche AR, Walsh EE, Formica MA, et al. Detection of respiratory viruses in sputum from adults by use of automated multiplex PCR. $J$ Clin Microbiol 2014;52:3590-6.

21 Xie X, Zhong Z, Zhao W, et al. Chest CT for typical coronavirus disease 2019 (COVID-19) pneumonia: relationship to negative RTPCR testing. Radiology 2020;296:E41-5.

22 Ai T, Yang Z, Hou H, et al. Correlation of chest CT and RT-PCR testing for coronavirus disease 2019 (COVID-19) in China: a report of 1014 cases. Radiology 2020;296:E32-40.

23 Kinloch NN, Ritchie G, Brumme CJ, et al. Suboptimal biological sampling as a probable cause of false-negative COVID-19 diagnostic test results. J Infect Dis 2020;222:899-902.

24 Patrucco F, Carriero A, Falaschi Z, et al. COVID-19 diagnosis in case of two negative nasopharyngeal swabs: association between chest CT and bronchoalveolar lavage results. Radiology 2021;298:E152-5.

25 Kozu Y, Gon Y, Maruoka S, et al. Protocadherin-1 is a glucocorticoidresponsive critical regulator of airway epithelial barrier function. BMC Pulm Med 2015;15:1-2 https://www.who.int/publications/i/item/ 10665-331501. Accessed 12/15/2020. doi:10.1186/s12890-0150078-z

26 Torrego A, Pajares V, Fernández-Arias C, et al. Bronchoscopy in patients with COVID-19 with invasive mechanical ventilation: a single-center experience. Am J Respir Crit Care Med 2020;202:284-7. 